\title{
SUPPRESSION OF THE MAGNETOELECTRIC HYSTERESIS IN A COMPOSITE LANGATATE-METGLAS STRUCTURE
}

\author{
Burdin D.A., Chashin D.V., Ekonomov N.A. Fetisov L.Y., and Fetisov Y.K. \\ Moscow Technological University (MIREA), 119454, Prosp. Vernadskogo, 78, Moscow, Russian Federation
}

\begin{abstract}
The suppression of the magnetoelectric effect hysteresis in a composite planar structure containing mechanically coupled layers of piezoelectric langatate and magnetostrictive amorphous alloy Metglas was observed and investigated. As the pumping magnetic field with frequency $\sim 84 \mathrm{kHz}$ was increased from 0.6 Oe up to 20 Oe, the coercive fields for the 1 st and 3rd voltage harmonics generated by the structure were decreased from $\sim 0.7$ Oe up to zero. The suppression of the magnetoelectric hysteresis will allow increasing of absolute accuracy of the magnetoelectric magnetic field sensors by $\sim 2$ orders of magnitude.
\end{abstract}

\section{INTRODUCTION}

Magnetoelectric (ME) effect is observed in composite planar structures consisting of ferromagnetic (FM) and piezoelectric (PE) layers. The effect exhbits itself as generation of electrical voltage by the structure under simultaneous action of a permanent $H$ and an alternating $h$ magnetic fields [1,2]. The magnetic field produces magnetostrictive deformation of the FM layer, this deformation is transmitted to the PE layer, and it generates electrical voltage due to piezoelectricity. The ME effect is considered as one of the most prospective tools for design of high-sensitivity magnetic field sensors operating at room temperature $[3,4]$.

However, it is well known that most of the ferromagnetic materials posses hysteretic behavior, that is the magnetization (as well as the magnetostrictive deformation) is an ambiguous function of the applied magnetic field. For materials with high magnetostriction, which are commonly used in composite ME structures, the coercive fields are $H_{\mathrm{C}} \sim 1$ Oe for amorphous alloy Metglas, $\sim 20$ Oe for nickel, and more that 50 Oe for rare-earth alloy Terfenol-D. The hysteretic phenomena result in big absolute measurement errors of $\mathrm{ME}$ magnetic field sensors or make necessary demagnetizing of the structure before each measurement, that strongly complicates design of the sensors. To the authors knowledge, these drawbacks of the ME magnetic field sensors have never been discussed up to now.

In this paper it is shown for the first time that hysteresis of ME effect in composite structures can be effectively suppressed by using pumping magnetic field of high amplitude. As it is known, an increase in the pumping field gives rise to a generation of higher harmonics of ME voltage [5]. So, the effect of hysteresis suppression was studied for the 1st and 3rd voltage harmonics, which can be used to design high-sensitivity magnetic field sensors [6,7]. This property of ME effect opens a possibility to elaborate high absolute accuracy censors for measuring permanent and alternating magnetic fields.

\section{EXPERIMENT}

A bilayer structure with dimensions of $20 \times 5 \mathrm{~mm}^{2}$, containing a layer of $\mathrm{x}$-cut single-crystal lanthanumgallium tantalite (LGT) of thickness $0.5 \mathrm{~mm}$ with metalized surfaces and a layer of amorphous alloy Metglas 2605S3A of thickness $20 \mu \mathrm{m}$ was used in measurements. The layers were mechanically connected to each other by low-temperature soldering. The Metglas was chosen because it is the most prospective material for the sensor fabrication, it has the smallest coercive field $H_{\mathrm{C}} \sim 1 \mathrm{Oe}$, rather high magnetostriction $\lambda_{\mathrm{S}} \approx 20 \cdot 10^{-6}$, and saturates in low magnetic fields $H_{\mathrm{s}} \sim 100 \mathrm{Oe}$.

The sample was placed in an electromagnetic coil which was fed by the tuned oscillator Agilent 33210a and created a pumping magnetic field $h \cos (2 \pi f t)$ with amplitude $h$ up to 20 Oe and frequency $f=0-100 \mathrm{kHz}$. The permanent bias magnetic field $H=0-50$ Oe was produced by the Helmholtz coils and was directed parallel to $h$ along the longest side of the structure. The magnetic fields were measured with a Lake Shore gauss meter with absolute accuracy $\sim 0.01$ Oe. Magnetoelectric voltage $u$ generated across electrodes of the LGT layer was measured with a lock-in amplifier SR 850.

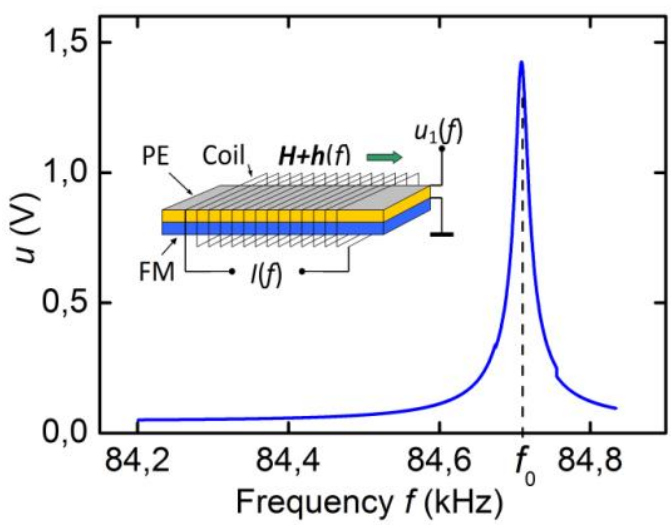

FIG. 1 Frequency responce of ME effect in a langatate-Metglas structure. The insert shows a schematic view of the composite structure.

\section{Y.K. Fetisov: fetisov@mirea.ru}


The dependences of $u$ as functions of excitation field frequency $f$ at fixed $H$ and $h$, and dependences of $u$ as functions of bias magnetic field $H$ for various pumping field amplitudes $h$ have been measured.

\section{RESULTS AND DISCUSSION}

Figure 1 shows frequency response of the structure, that is dependence of generated voltage $u$ on the excitation magnetic field frequency $f$ for fixed $H=1$ Oe and alternating field $h=0.08$ Oe. One can see a peak with height of $1.42 \mathrm{~V}$ and quality factor of $Q \approx 5100$ in the vicinity of the frequency $f_{0}=84.71 \mathrm{kHz}$. The peak corresponds to excitation of longitudinal acoustic oscillations in the structure which results in an increase in the deformation and "amplification" of the ME effect. For the given structure the ME coefficient $\alpha_{E}=u /(b h)$, where $b$ is the FE layer thickness, was equal to $\sim 12.5 \mathrm{~V} /(\mathrm{cm} \cdot \mathrm{Oe})$ far away of the resonance and $\sim 355 \mathrm{~V} /(\mathrm{cm} \cdot \mathrm{Oe})$ at the resonance frequency, that coincide with data of [8].

In order to figure out the value of the hysteretic field for the described structure, the magnetization loop was measured (at Department of Magnetism, MSU) using the Lake Shore vibration sample magnetometer. The loop is shown in Fig. 2. One can see that the loop is symmetric with respect to the origin of coordinates and the sample saturates in the fields $H_{\mathrm{S}} \sim 100$ Oe. The insert of Fig. 2 shows initial part of the loop in expanded scale. The coercive field for Metglas is $H_{\mathrm{C}} \approx 1 \mathrm{Oe}$. As in the low field region the relation $\lambda \sim M^{2}$ is true, the coercive field for the dependence $\lambda(H)$ is also equal to $1 \mathrm{Oe}$.

In order to observe the hysteresis, the dependences of generated voltage $u$ on the field $H$ for fixed signal frequency $f$ and different amplitudes of the pumping field $h$ have been measured. To avoid changes in the generated voltage due to a shift in the resonance frequency caused by $H$ variation (so called $\Delta E$ effect), all the measurements were carried out on the slope of the resonance curve (see Fig.1) at the frequency $84.6 \mathrm{kHz}$.

First, the magnetic field dependences for the $1 \mathrm{st}$ voltage harmonic have been measured. The structure was excited by magnetic field with a frequency $84.6 \mathrm{kHz}$ and the signal was registered at the same frequency. Figure 3 shows dependences of the 1 st harmonic amplitude $u_{1}$ on the field $H$ for pumping field amplitudes $h=0.6$ Oe and

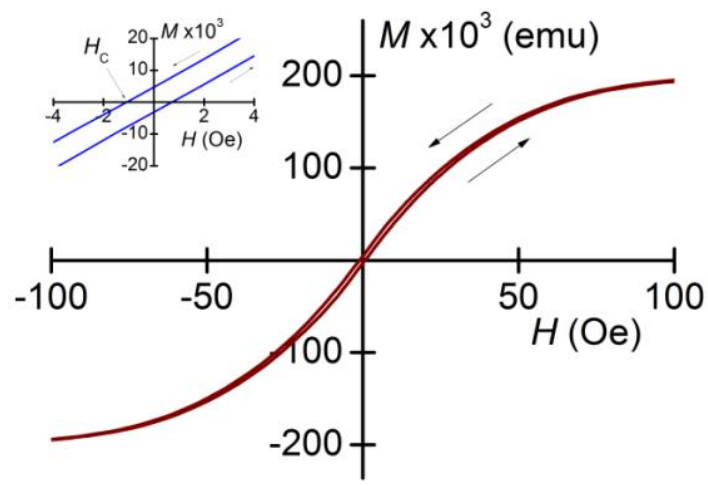

FIG. 2 Magnetization curve of the Metglas layer. The insert shows initial part of the curve in expanded scale.
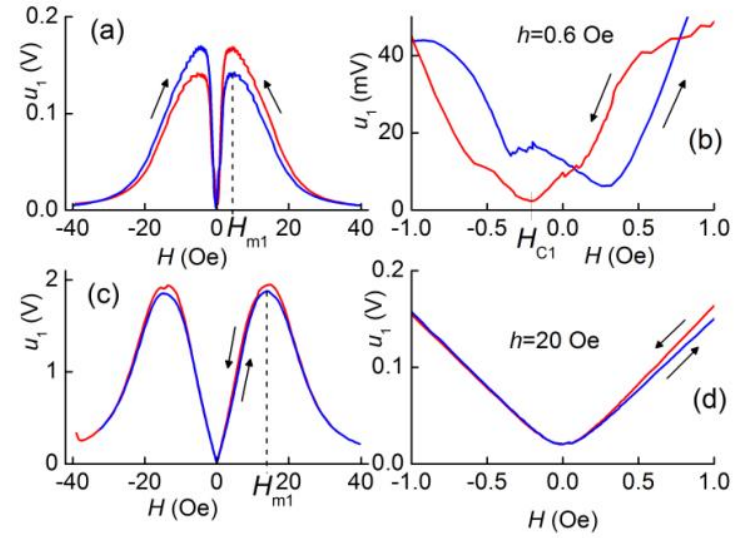

FIG. 3 Dependence of the 1 st harmonic of the ME voltage $u_{1}$ on permanent magnetic field $H$ for different amplitudes of the pumping field $h=0.6$ Oe and $h=20$ Oe.

$h=20$ Oe. It is seen, that for low pumping field $h=0.6$ Oe (Fig.3a), $u_{1}$ first increases with the field increase, reaches a maximum at $H_{\mathrm{m} 1} \approx 4.2 \mathrm{Oe}$, and then drops to zero. For high pumping field amplitude $h=20$ Oe (Fig.3c), the dependence has similar shape, but the maximum takes place in bigger field $H_{\mathrm{m} 1} \approx 13.7 \mathrm{Oe}$. It is seen in expanded scale (Figs. $3 \mathrm{~b}$ and $3 \mathrm{~d}$ ), that for low pumping field the hysteresis on the dependence $u_{1}(H)$ takes place for increasing and decreasing fields. The value of the coercive field is $H_{\mathrm{C} 1} \approx 0.25$ Oe. For high pumping field $h=20$ Oe (see Fig. 3d) the hysteresis vanishes. Note, that $u_{1}$ is not equal to zero at $H=0$ for the 1 st harmonic. This is due to the effect of direct electromagnetic interference at a large amplitude of the pumping field.

After that similar measurements have been carried out for the 3rd ME voltage harmonic. The structure was excited by magnetic field with frequency of $28.2 \mathrm{kHz}$ and generated signal at the frequency $84.6 \mathrm{kHz}$ was registered. Results of the measurements are presented in Fig. 4.

It is seen from Fig.4, that the dependence $u_{3}(H)$ has more complicated shape and contains three peaks as the field $H$ increases from zero up to 40 Oe. For low pumping field amplitude $h=4$ Oe (Fig. 4a) the first maximum is reached in the field $H_{\mathrm{m} 3} \approx 1.3 \mathrm{Oe}$, while for high pumping field (Fig. 4c) it is reached in the field $H_{\mathrm{m} 3}$

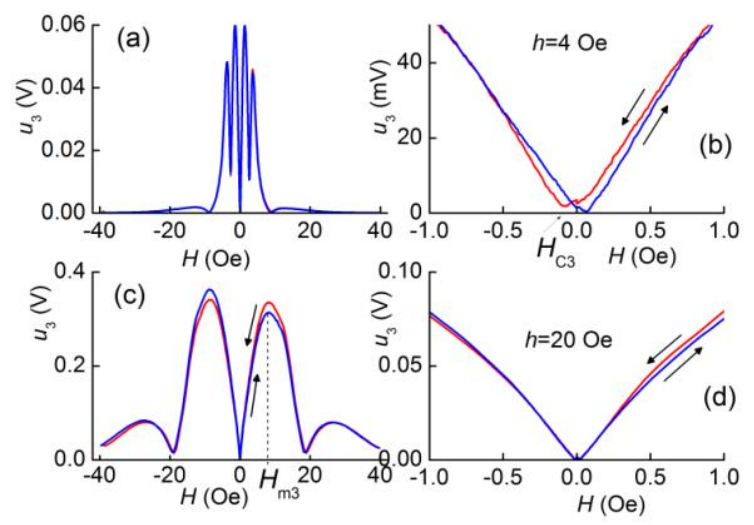

FIG. 4 Dependence of the 3rd harmonic of the ME voltage $u_{3}$ on permanent magnetic field $H$ for different amplitudes of the pumping field $h=4$ Oe and $h=20$ Oe. 
$\approx 8.2$ Oe. It is seen in expended scale (Figs. $4 \mathrm{~b}$ and $4 \mathrm{~d}$ ), that the dependence $u_{3}(H)$ also has a hysteresis. For low pumping field $h=4 \mathrm{Oe}$, the coercive field is $H_{\mathrm{C} 3} \approx 0.07$ Oe. For high pumping field $h=20$ Oe the hysteresis is completely vanishes and $u_{3}=0$ in the field absence $H=0$. The direct electromagnetic interference with pumping frequency does not contribute to the amplitude of the 3rd harmonic.

Using measurements similar to those shown in Fig. 3 and Fig. 4, one can plot the dependences of characteristic fields $H_{\mathrm{m} 1}$ and $H_{\mathrm{m} 3}$, corresponding to the first maximum of the 1 st and the 3 rd harmonics, on the pumping field $h$. It follows from Fig. 5, that these characteristic fields remain constant in the pumping field region $h<4$ Oe, and then increase as the pumping field growths. Thus, the operation range of the ME magnetic field sensor expands with an increase of $h$, which coincides with conclusions of [7].

Figure 5 shows dependences of coercive fields $H_{\mathrm{C} 1}$ and $H_{\mathrm{C} 3}$ for the 1 st and 3 rd harmonics on the pumping field $h$, drawn using the data which are similar to those given in Fig. 3 and Fig. 4. It is seen, that the coercive field for both harmonics falls down with an increase of $h$

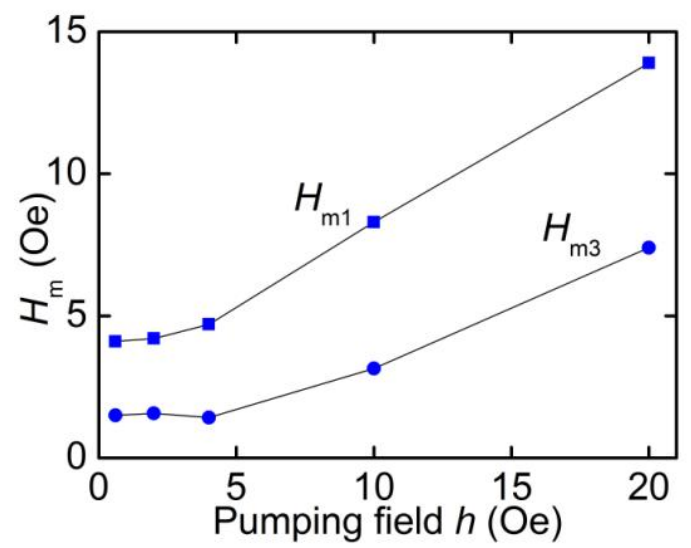

FIG. 5 Dependences of the characteristic fields $H_{\mathrm{m} 1}$ and $H_{\mathrm{m} 3}$, corresponding to maxima of the $1 \mathrm{st}$ and $3 \mathrm{rd} \mathrm{ME}$ voltage harmonics, on the pumping field $h$.

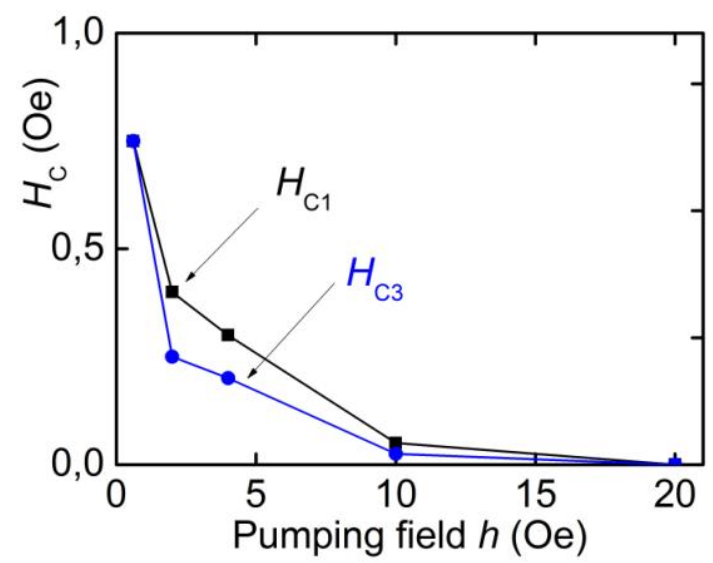

FIG. 6 Dependences of the coercive fields $H_{\mathrm{C} 1}$ and $H_{\mathrm{C} 3}$ for the 1st and 3rd ME voltage harmonics on the pumping field $h$. and it turns out to zero at $h \approx 20$ Oe. Inaccuracy in determining minima of curves in Fig. 3 and Fig. 4 does not allow to draw these curves more accurately and find an analytical formula for the hysteresis suppression.

Theoretical simulation showed that the effect of ME hysteresis suppression is due to a decrease in the relative influence of the constant field on the asymmetry of the minor magnetostriction loop of a ferromagnetic with increasing amplitude of the alternating field. Complete suppression of the hysteresis is achieved when amplitude of the pumping field turns out comparable with the saturation field of the ferromagnetic layer of the structure.

\section{CONCLUSION}

Thus, the effect of the hysteresis suppression in the field dependence of the ME voltage harmonics generated by the langatate-Metglas structure was experimentally observed. The effect will allow a $\sim 2$ order of magnitude enhancement in the accuracy of the absolute measurements of the ME magnetic field sensors.

The research was supported by the Russian Foundation for Basic Research (grant No 17-12-01435).

\section{References:}

1. C.-W. Nan, M.I. Bichurin, S. Dong, D. Viehland, G. Srinivasan, J. Appl. Phys., 103, p. 031101 (2008).

2. H. Palneedi, V. Annapureddy, S. Priya, J. Ryu, Actuators, 5, No 9, p. 1-31 (2016).

3. M.M. Vopson, Critical Rev. in Solid States and Mater. Sci. 40, p. 1-28 (2014).

4. Y. Wang, J. Le, D. Viehland, Materials Today, 17, p. $269-275$ (2014).

5. K.E. Kamentsev, Y.K. Fetisov, and G. Srinivasan, APL, 89, p. 142510 (2006).

6. D.A. Burdin, D.V. Chashin, N.A. Ekonomov, Y.K. Fetisov, A.A. Stashkevich, JMMM, 405, p. 244-248 (2016).

7. D.A. Burdin, D.V. Chashin, N.A. Ekonomov, L.Y. Fetisov, Y.K. Fetisov, M. Chamonin, J. Phys. D: Appl. Phys., 49, p. 375002 (2016).

8. G. Sreenevasulu, L.Y. Fetisov, Y.K. Fetisov, and G. Srinivasan, APL, 100, p. 052901 (2012). 